\title{
Measuring the solvent quality of water for disordered proteins from a single SAXS measurement.
}

Joshua A Riback ${ }^{1}$, Micayla A Bowman ${ }^{2}$, Adam Zmyslowski ${ }^{3}$, Catherine R Knoverek ${ }^{2}$, John M Jumper $^{3,4}$, James Hinshaw ${ }^{4}$, Emily B Kaye ${ }^{2}$, Karl F Freed ${ }^{4}$, Patricia L Clark*2, Tobin R Sosnick*3,5

${ }^{1}$ Graduate Program in the Biophysical Sciences

${ }^{3}$ Department of Biochemistry and Molecular Biology

${ }^{4}$ Department of Chemistry

${ }^{5}$ Institute for Biophysical Dynamics

University of Chicago, Chicago, IL 60637

${ }^{2}$ Department of Chemistry and Biochemistry

University of Notre Dame

Notre Dame, IN 46556

Proteins adopt non-native geometries during their synthesis, folding, transport, and turnover and a significant fraction of the proteome is intrinsically disordered. The characterization of disordered proteins is challenging in part due to difficulties in the interpretation of techniques including SAXS and FRET. We have developed a method to extract the dimensions and solvent quality of a polymer from a single SAXS measurement. We apply it to intrinsically disordered proteins (IDPs), finding them to be expanded in water, which behaves as a good solvent. Additionally, we use our methodology to demonstrate that labeling with Alexa488 causes the IDP to undergo significant dye-induced contraction. While advances in methodology of SAXS and FRET narrowed the discrepancy between them on the dimensions of IDPs, our newer results demonstrate that the addition of dyes influences the properties of IDPs and is likely the primary origin of the remaining discrepancy between the methods. Recent studies have suggested that FRET and SAXS results can be reconciled if SAXS reports on $\mathrm{R}_{\mathrm{g}}$ while remaining relatively insensitive to Rend-to-end, essentially decoupling the two. To the contrary, our analysis finds that the entire SAXS profile is sensitive to the end-to-end distance. More broadly, our simulations define a route to characterize deviations from homopolymeric behavior inherent in disordered proteins. 